\title{
Inhaltsverzeichnis Sonderband: Holz im Hochbau
}

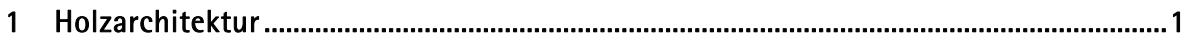

$1 \mid 1 \quad$ Zur Geschichte des Holzbaus .................................................................................................... 2

$1 \mid 2$ Typologien und Systeme, Werkzeuge der Planung …….................................................. 9

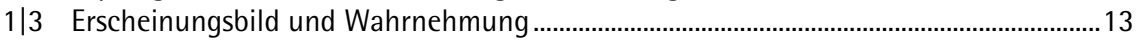

$1 \mid 4 \quad$ Vorfertigung und Serienfertigung .................................................................................. 15

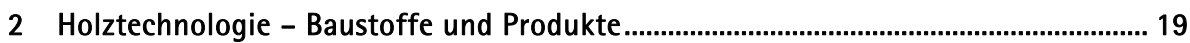

2|1 Aufbau und Struktur des (Bau-)Holzes............................................................................... 19

$2|1| 1 \quad$ Rohstofflieferant Wald ...................................................................................... 19

$2|1| 2$ Makroskopische Struktur, Wuchseigenschaften, Anisotropie..............................20

$2|1| 3 \quad$ Mikroskopische Struktur ........................................................................................21

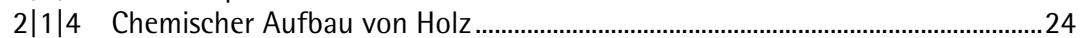

2|1|5 Heimische Hölzer für den konstruktiven Hochbau ................................................24

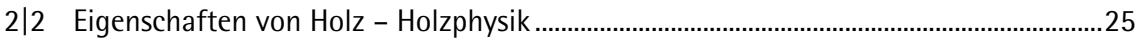

$2|2| 1 \quad$ Grundlegende (physikalische) Eigenschaften .....................................................25

$2|2| 1 \mid 1 \quad$ Dichte (Rohdichte)..................................................................................................2

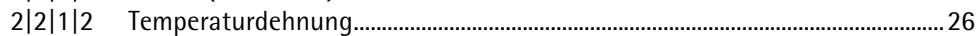

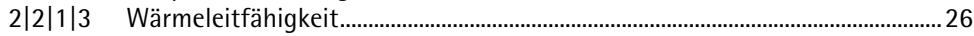

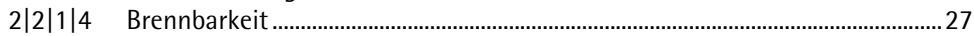

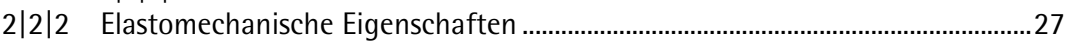

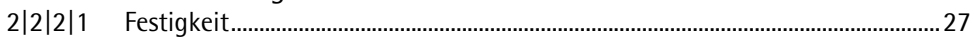

$2|2| 2 \mid 2 \quad$ Elastizitäts- und Schubmodul, Kriechverhalten.....................................................27

$2|2| 2 \mid 3 \quad$ Zusammenstellung elastomechanische Eigenschaften.........................................28

$2|2| 3 \quad$ Feuchtetechnische Eigenschaften und ihr Einfluss............................................28

$2|2| 3 \mid 1 \quad$ Wassersättigung, Fasersättigung, Darrzustand ....................................................28

$2|2| 3 \mid 2 \quad$ Hygroskopisches Gleichgewicht ...........................................................................29

$2|2| 3 \mid 3 \quad$ Luftfeuchtigkeit und Holzfeuchtigkeit im Fasersättigungsbereich....................30

$2|2| 3 \mid 4 \quad$ Natürliche Dauerhaftigkeit........................................................................................30

$2|2| 4 \quad$ Schwinden und Quellen ........................................................................................

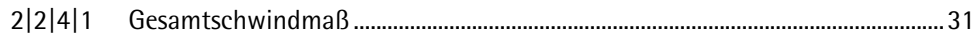

$2|2| 4 \mid 2 \quad$ Differenzielles Schwind- und Quellmaß..............................................................32

$2|2| 4 \mid 3 \quad$ Dimensionsstabilisierende Maßnahmen ................................................................32

$2|2| 5 \quad$ Bestimmung der Holzfeuchte..................................................................................33

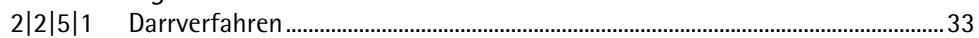

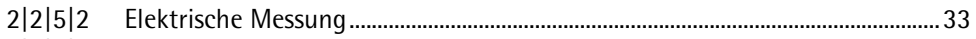

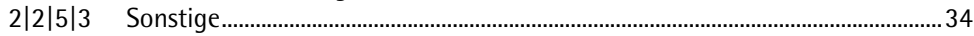

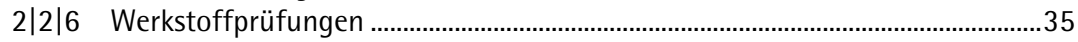

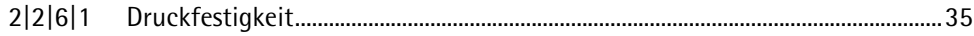

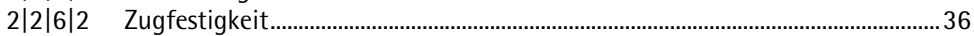

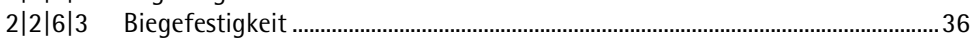

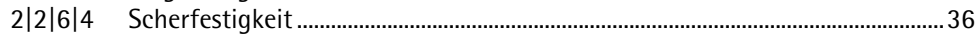

$2|2| 6 \mid 5 \quad$ Nagel- und Schraubenausziehwiderstand ...........................................................37

$2|2| 6 \mid 6 \quad$ Verklebungen - Eignung von Klebeverbindungen.................................................37

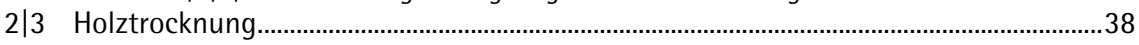

$2|3| 1 \quad$ Natürliche Trocknung / Lufttrocknung...............................................................38

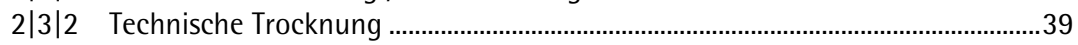

$2|3| 2 \mid 1 \quad$ Grundprinzipien der Konvektionstrocknung........................................................ 39

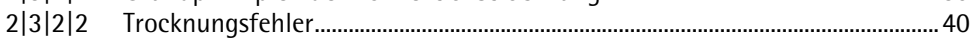

$2|3| 3 \quad$ Gebrauchsfeuchtigkeit ................................................................................... 42

2|4 Holzschädlinge und Holzschutz ................................................................................. 42

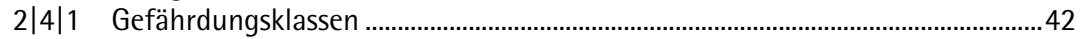

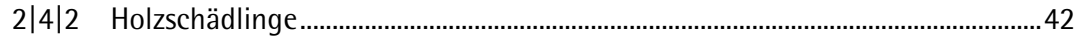

$2|4| 2 \mid 1 \quad$ Holzverfärbende Pilze.............................................................................................. 43

$2|4| 2 \mid 2 \quad$ Holzzerstörende Pilze.......................................................................................... 43

$2|4| 2 \mid 3 \quad$ Tierische Schädlinge (Insekten) ................................................................................ 44 
2|4|3 Grundprinzip Holzschutz - vorbeugende Maßnahmen.......................................45

$2|4| 3 \mid 1 \quad$ Baulich-konstruktiver Holzschutz ……………...................................................... 45

$2|4| 3 \mid 2 \quad$ Chemischer Holzschutz ...........................................................................................4

$2|4| 4$ Bekämpfung von Holzschädlingen ..................................................................... 47

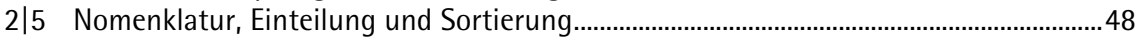

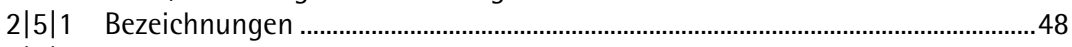

$2|5| 2 \quad$ Einteilung und Sortierung von Holz.................................................................. 49

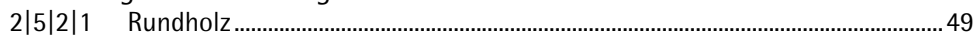

$2|5| 2 \mid 2 \quad$ Schnittholz gemäß der Festigkeit ....................................................................... 50

$2|5| 2 \mid 3 \quad$ Schnittholz gemäß der Oberfläche …………………………………………….......51

2|6 Holz und Holzwerkstoffe ..............................................................................................52

$2|6| 1$ Produkte vorwiegend für die Primärkonstruktion ...................................................53

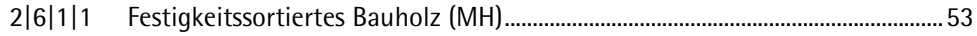

$2|6| 1 \mid 2 \quad$ Keilgezinktes Konstruktionsvollholz (KVH) .............................................................54

$2|6| 1 \mid 3 \quad$ Balkenschichtholz ..................................................................................................5

$2|6| 1 \mid 4 \quad$ Brettschichtholz (BSH) ...................................................................................... 55

$2|6| 1 \mid 5 \quad$ Kreuzholzbalken (Kreuzholz, Kreuzbalken)..........................................................56

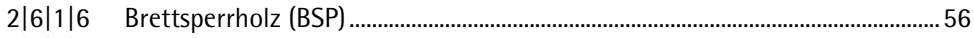

$2|6| 1 \mid 7 \quad$ Furnierschichtholz ................................................................................................. 57

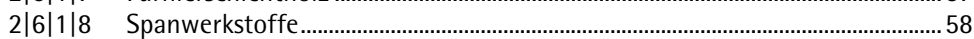

$2|6| 2$ Produkte vorwiegend zur Aussteifung und Beplankung....................................58

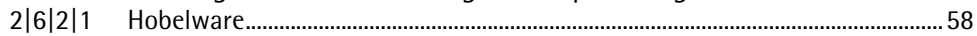

$2|6| 2 \mid 2 \quad$ Massivholzplatte breitenverleimt ……………………………………………………....59

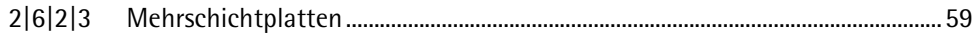

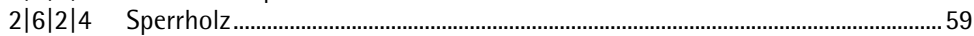

$2|6| 2 \mid 5 \quad$ OSB-Platten (Oriented Strand Board) …………………………………………....59

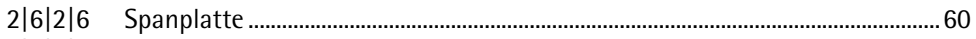

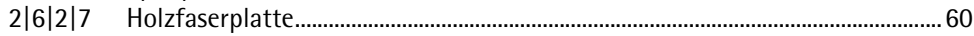

$2|6| 3$ Holzweichfaser-Putzträgerplatte …………....................................................61

$2 \mid 7$ Sonstige gängige Baustoffe für den Holzbau ..................................................................61

$2|7| 1$ Dämmstoffe .................................................................................................61

$2|7| 1 \mid 1 \quad$ Holzfaserdämmstoffe ..................................................................................................61

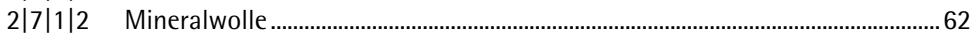

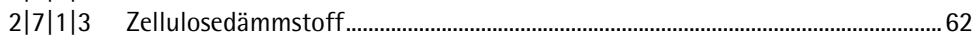

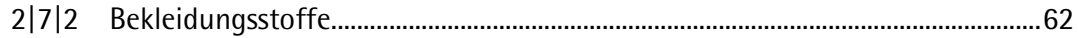

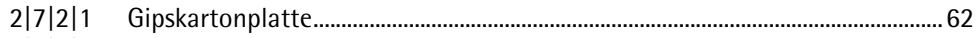

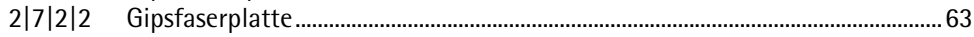

$2|7| 2 \mid 3 \quad$ Zementgebundene, bewehrte Leichtbetonplatte ...................................................63

$2|7| 2 \mid 4 \quad$ Holzwolle-Leichtbauplatte (HWL)...........................................................................63

$2|7| 3 \quad$ Folien/Abdichtungen.....................................................................................................63

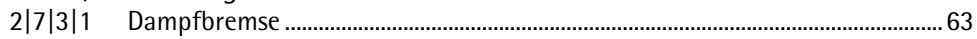

$2|7| 3 \mid 2 \quad$ Diffusionsoffene Folie ………………………………………………………………......

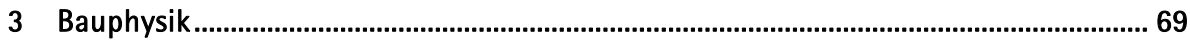

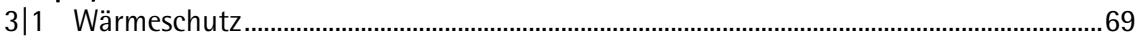

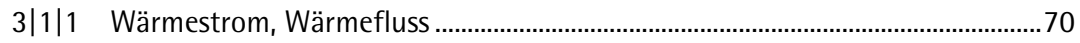

$3|1| 2 \quad$ U-Wert .......................................................................................................... 72

$3|1| 2 \mid 1 \quad$ Berechnung homogene Bauteile.............................................................................. 72

$3|1| 2 \mid 2 \quad$ Berechnung inhomogene Bauteile..........................................................................

$3|1| 2 \mid 3 \quad$ Vorabschätzung des U-Wertes ………………………………………………….....75

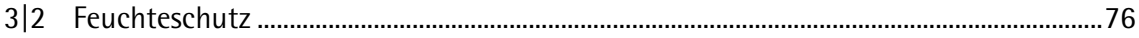

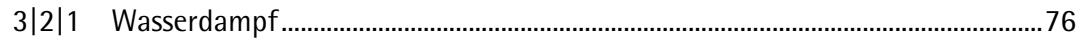

$3|2| 1 \mid 1 \quad$ Wasserdampf-Sättigungsdruck ..........................................................................76

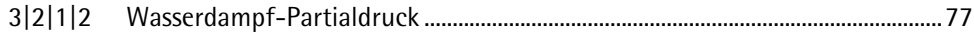

$3|2| 1 \mid 3 \quad$ Relative Luftfeuchtigkeit............................................................................................

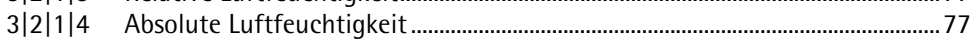

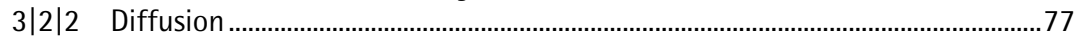

$3|2| 2 \mid 1 \quad$ Wasserdampf-Diffusionswiderstandszahl..........................................................78

$3|2| 2 \mid 2 \quad$ Wasserdampf-diffusionsäquivalente Luftschichtdicke...........................................78 


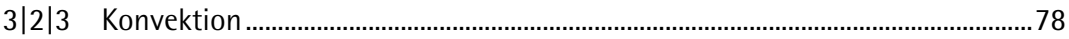

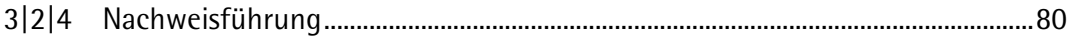

$3|2| 4 \mid 1 \quad$ Konstruktionen ohne weitere Nachweise ............................................................. 80

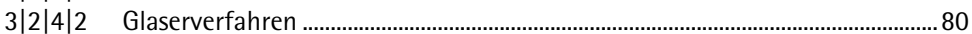

$3|2| 4 \mid 3 \quad$ Hygrothermische Simulation .............................................................................

$3|2| 5$ Baupraktische Empfehlungen bei diffusionsoffenen Konstruktionen ...............81

3|2|6 Maßnahmen bei Konstruktionen mit außenseitig hohen Sd-Werten .................81

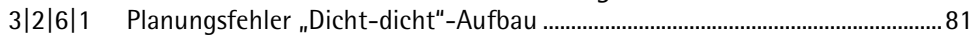

$3|2| 6 \mid 2 \quad$ Einflussfaktoren .............................................................................................................. 82

$3|2| 6 \mid 3 \quad$ Nachweispyramide....................................................................................................... 82

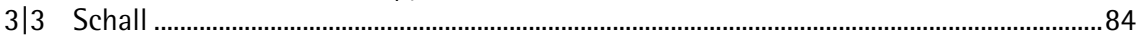

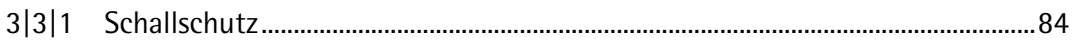

$3|3| 1 \mid 1 \quad$ Grundlagen der Akustik ........................................................................................... 85

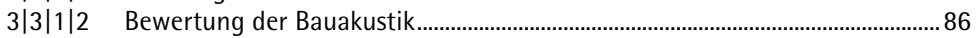

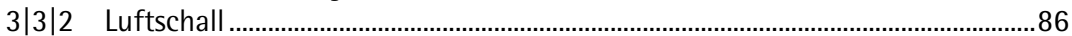

$3|3| 2 \mid 1 \quad$ Luftschalldämmung einschaliger, mineralischer, massiver Bauteile..................8 88

$3|3| 2 \mid 2 \quad$ Luftschalldämmung einschaliger, massiver, aber leichter Bauteile....................89

$3|3| 2 \mid 3 \quad$ Luftschalldämmung mehrschaliger, leichter Bauteile ........................................90

$3|3| 2 \mid 4 \quad$ Vorsatzschalen zur akustischen Verbesserung von Bauteilen ............................92

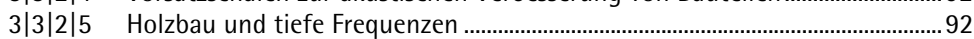

$3|3| 3 \quad$ Körperschall ..............................................................................................................93

$3|3| 3 \mid 1 \quad$ Reduktion von Körperschall....................................................................................94

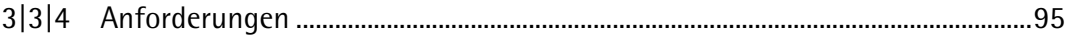

$3|3| 4 \mid 1 \quad$ Anforderungen an Außenbauteile.......................................................................95

$3|3| 4 \mid 2$ Anforderungen an Innenbauteile …………………………...............................96

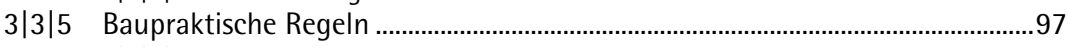

$3|3| 5 \mid 1$ Außenwände ...........................................................................................................97

$3|3| 5 \mid 2$ Trennwände................................................................................................................99

$3|3| 5 \mid 3 \quad$ Trenndecken .............................................................................................................99

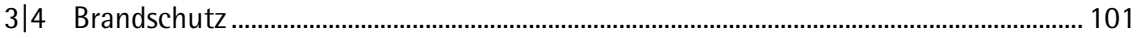

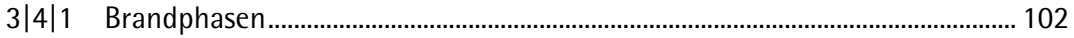

$3|4| 2 \quad$ Brandverhalten von Baustoffen ....................................................................... 103

$3|4| 3 \quad$ Abbrand von Holz .............................................................................................. 104

$3|4| 3 \mid 1 \quad$ Abbrandraten gemäß ÖNORM EN 1995-1-2 [196] ..........................................105

$3|4| 3 \mid 2 \quad$ Abbrandrate für Brettsperrholz............................................................................105

$3|4| 3 \mid 3 \quad$ Varianten der Dimensionierung durch Beplankung …………………………....106

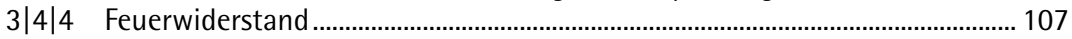

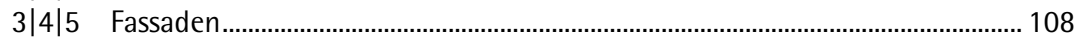

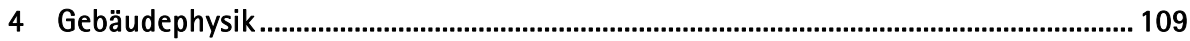

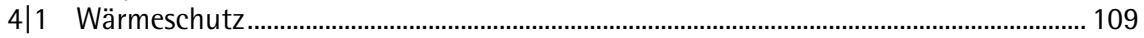

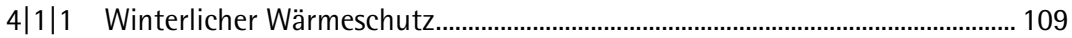

$4|1| 2$ Luft- und Winddichtheit......................................................................................... 109

$4|1| 3 \quad$ Sommertauglichkeit ................................................................................................. 110

$4|1| 3 \mid 1 \quad$ Luftwechselrate .....................................................................................................110

$4|1| 3 \mid 2 \quad$ Solare Einträge, Sonnenschutz...........................................................................112

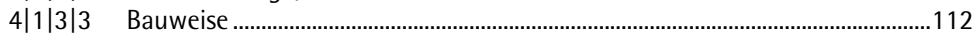

$4|1| 3 \mid 4 \quad$ Fensterdiagramme ...................................................................................................114

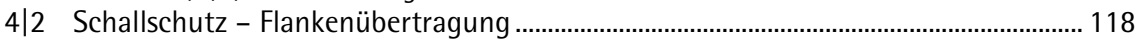

$4|2| 1 \quad$ Durchlaufende Decken ......................................................................................... 119

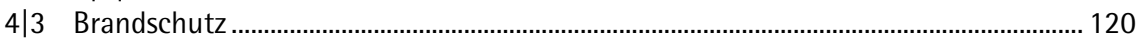

$4|3| 1 \quad$ Anforderungen ........................................................................................... 120

$4|3| 1 \mid 1 \quad$ Fassaden ..................................................................................................................... 123

$4|3| 1 \mid 2 \quad$ Abweichungen von Anforderungen der OIB-Richtlinien..................................124

$4|3| 2$ Durchdringungen und Abschottungen.............................................................. 124

$4|3| 2 \mid 1 \quad$ Vertikale Verteilung...……………………………………………………………124

$4|3| 2 \mid 2 \quad$ Horizontale Verteilung ......................................................................................126

$4|3| 2 \mid 3 \quad$ Abschottungssysteme durch Brandabschnitte....................................................126 


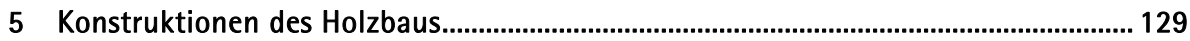

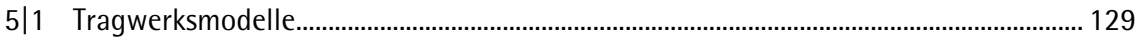

$5|1| 1 \quad$ Gebäudestabilität................................................................................................ 130

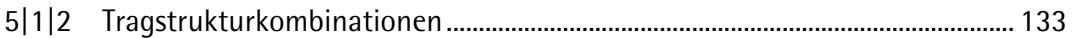

$5 \mid 2$ Bauweisen, Wand- und Deckenkonstruktionen ............................................................... 134

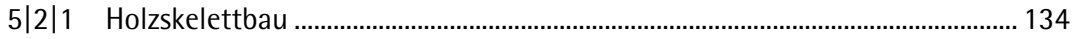

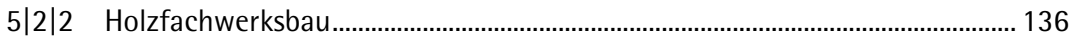

$5|2| 3$ Holzrahmenbau, Holzriegelbau......................................................................... 136

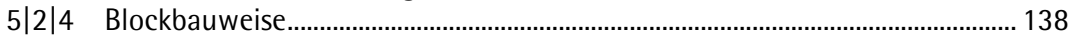

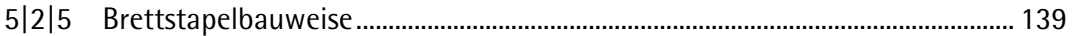

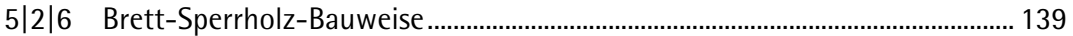

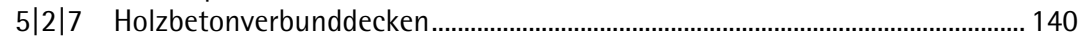

5|2|8 Kontaktfugen Brettsperrholzplattenelemente .................................................... 142

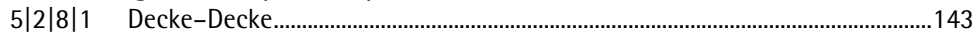

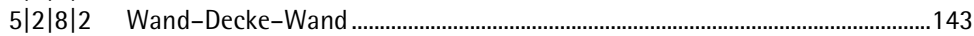

$5|2| 8 \mid 3 \quad$ Eckstoß Wand-Wand ................................................................................................144

$5|2| 8 \mid 4 \quad$ Wand-Fundierung ……………………………………………………………………14

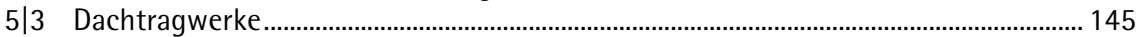

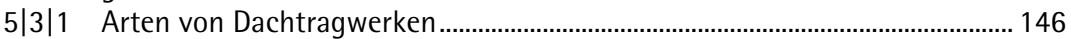

$5|3| 2 \quad$ Sparrendächer ......................................................................................................... 146

$5|3| 3$ Pfettendächer .................................................................................................. 147

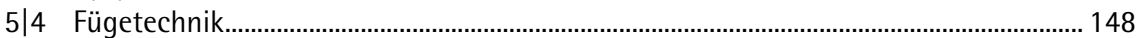

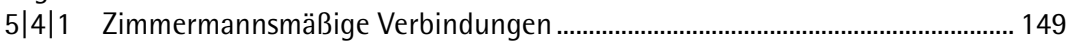

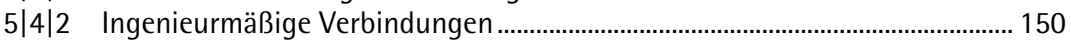

$5|4| 2 \mid 1 \quad$ Stiftförmige Verbindungsmittel ...........................................................................150

$5|4| 2 \mid 2 \quad$ Flächenhaft wirkende Verbindungsmittel.............................................................154

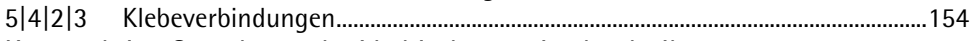

$5|4| 3 \quad$ Konstruktive Gestaltung der Verbindungsmitteltechnik.................................. 155

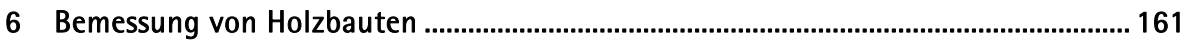

$6 \mid 1$ Bemessungsgrundlagen .............................................................................................. 162

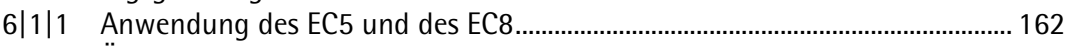

6|1|2 ÖNORMEN EN 1990 - CC - Klassen .................................................................. 163

6|1|3 ÖNORM EN 1990 - Überwachungsmaßnahmen ............................................... 164

$6|1| 3 \mid 1 \quad$ Überwachungsmaßnahmen bei der Planung (DSL) ...........................................164

$6|1| 3 \mid 2$ Überwachungsmaßnahmen bei der Herstellung (IL) ..........................................164

6|1|4 Sicherheitskonzept ............................................................................................. 165

6|2 Einwirkungskombinationen - Bemessungswerte............................................................ 166

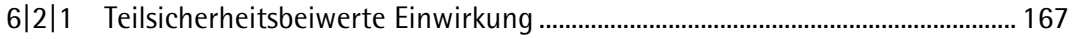

$6|2| 2$ Bemessungswerte der Einwirkungen................................................................ 167

$6|2| 2 \mid 1 \quad$ Ständige oder vorübergehende Bemessungssituationen

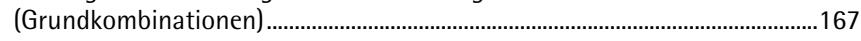

$6|2| 2 \mid 2 \quad$ Außergewöhnliche Bemessungssituationen.......................................................168

$6|2| 2 \mid 3 \quad$ Bemessungssituationen bei Erdbebeneinwirkungen .............................................168

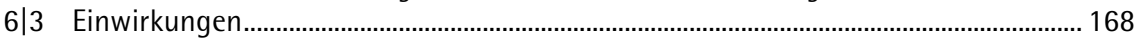

$6|3| 1 \quad$ Eigengewichtslasten .......................................................................................... 168

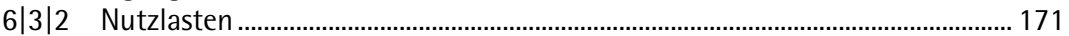

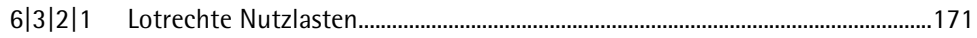

$6|3| 2 \mid 2 \quad$ Horizontale Nutzlasten ......................................................................................173

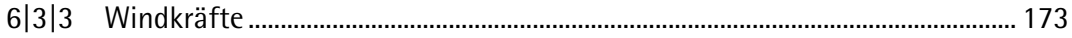

$6|3| 4$ Schneelasten....................................................................................................... 178

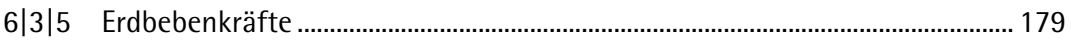

6|3|6 Außergewöhnliche Einwirkungen ................................................................. 183

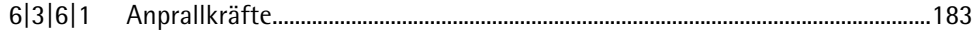

$6|3| 6 \mid 2$ Zwänge ..........................................................................................................183

6|4 Widerstände nach ÖNORM EN 1995 - EC 5................................................................... 183

$6|4| 1 \quad$ Charakteristische Festigkeiten............................................................................. 184 


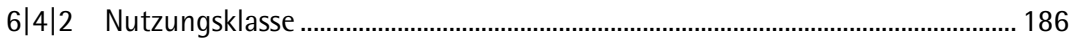

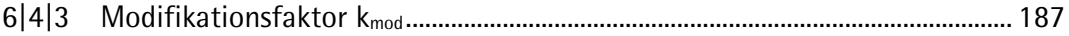

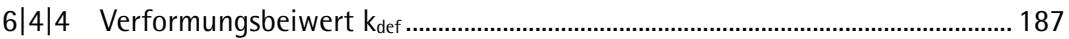

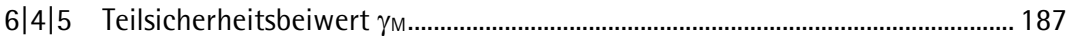

6|4|6 Bemessungswerte der Festigkeitseigenschaften ................................................ 188

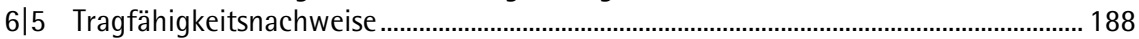

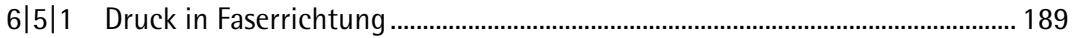

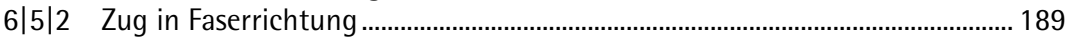

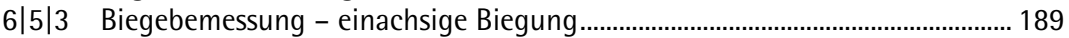

$6|5| 4$ Biegebemessung - zweiachsige Biegung ............................................................ 190

6|5|5 Biegung und Normalkraft .............................................................................. 191

$6|5| 5 \mid 1 \quad$ Biegung - zweiachsig und Zug........................................................................191

6|5|5|2 Biegung - zweiachsig und Druck......................................................................192

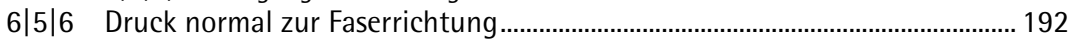

6|5|7 Druck unter einem Winkel zur Faser ................................................................... 193

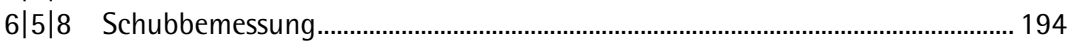

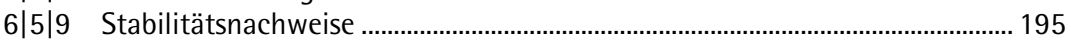

6|5|9|1 Knicken von Druckstäben ....................................................................................195

$6|5| 9 \mid 2 \quad$ Kippen von Biegestäben ..................................................................................199

$6|5| 9 \mid 3 \quad$ Knick- und Kippbeanspruchung.......................................................................2.

6|5|10 Holzbetonverbunddecken .................................................................................... 204

$6|5| 10 \mid 1$ Bemessung - Nachweiskonzept ..........................................................................204

$6|5| 10 \mid 2$ Bedeutung und Grenzen des Gamma-Verfahrens .............................................206

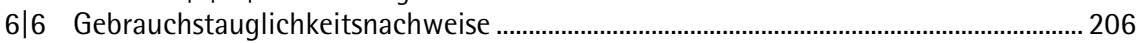

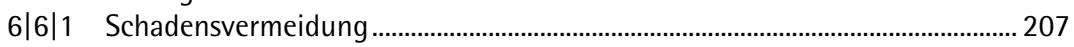

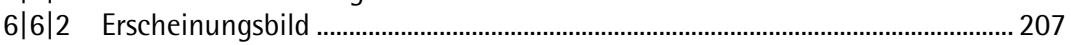

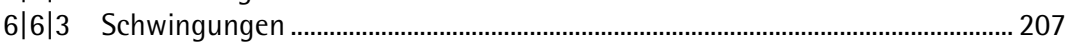

$6|6| 3 \mid 1 \quad$ Frequenzkriterium...............................................................................................208

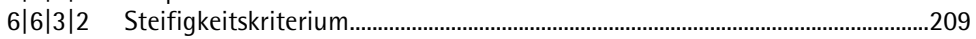

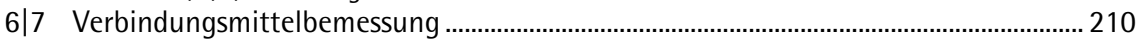

$6|7| 1 \quad$ Zimmermannsmäßige Verbindungen .................................................................. 210

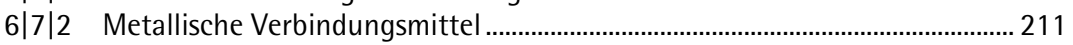

$6|7| 2 \mid 1 \quad$ Nägel .......................................................................................................................216

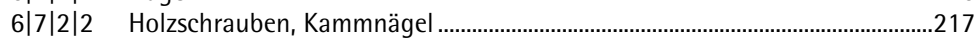

$6|7| 2 \mid 3 \quad$ Stabdübel, Schrauben- und Passbolzen ............................................................22

6|8 Konstruktionsgrundsätze und Bemessung von Aussteifungselementen ....................... 224

$6|8| 1 \quad$ Wandscheiben .................................................................................................... 224

$6|8| 2$ Dach- und Deckenscheiben ................................................................................. 228

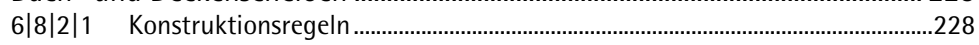

$6|8| 2 \mid 2 \quad$ Kräftespiel und Schubfluss in Deckenscheiben....................................................229

$6|8| 2 \mid 3 \quad$ Regelungen für vereinfachten Nachweis..........................................................220

$6|8| 3$ Bemessungsnachweise des Schubflusses bei Holztafeln ................................... 230

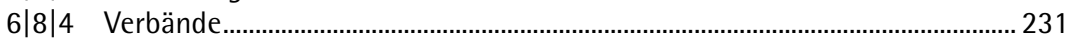

$6|8| 4 \mid 1 \quad$ Stabilisierungskraft eines beanspruchten Einzelbauteils.................................231

$6|8| 4 \mid 2$ Aussteifung von Trägern und Fachwerken ........................................................232

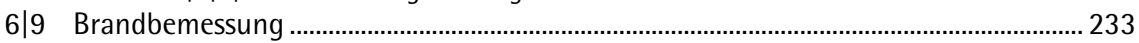

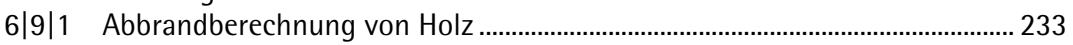

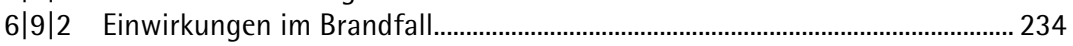

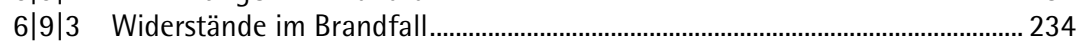

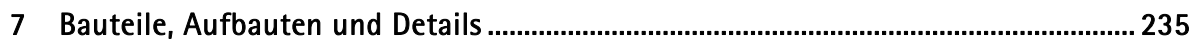

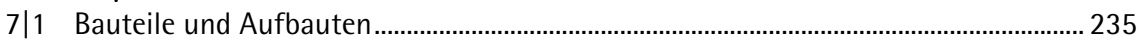

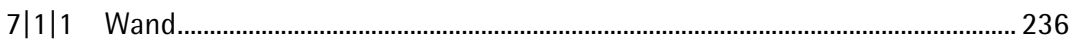

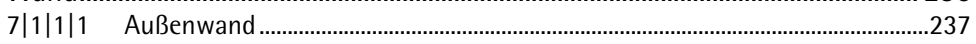

$7|1| 1 \mid 2 \quad$ Innenwand .......................................................................................................241

$7|1| 1 \mid 3 \quad$ Trennwand / Wand an Grundgrenze (Brandwand) .............................................243

$7|1| 2 \quad$ Decke ........................................................................................................................ 245 
7|1|2|1 Geschoßdecke innerhalb Wohn-/Betriebseinheit..............................................245

$7|1| 2 \mid 2 \quad$ Trenndecke zwischen Wohn-/Betriebseinheiten.................................................249

$7|1| 2 \mid 3 \quad$ Decke gegen ungedämmten Dachraum ...........................................................250

7|1|2|4 Kellerdecke........................................................................................................251

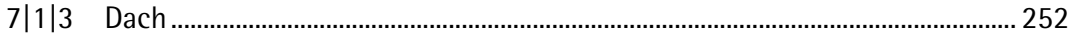

$7|1| 3 \mid 1 \quad$ Steildach ........................................................................................................253

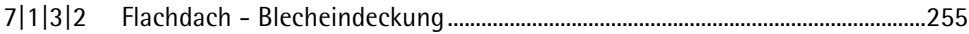

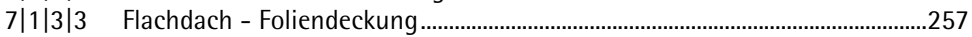

7|1|4 Besonderheiten der Fassadenbekleidung/-verkleidung ..................................... 259

7| $1|4| 1 \quad$ Holzfassaden.......................................................................................................25

$7|1| 4 \mid 2 \quad$ Geputzte Fassaden - Holzfaser-Wärmedämmverbundsystem..........................262

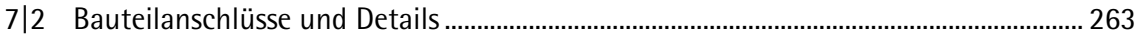

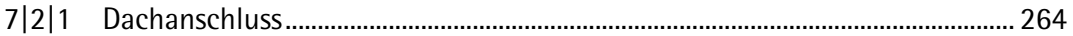

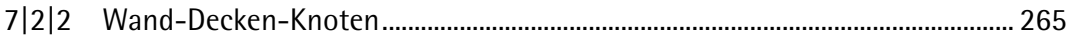

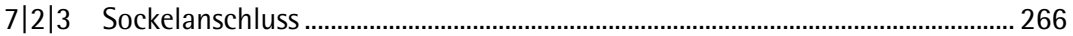

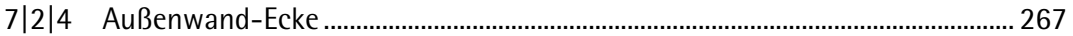

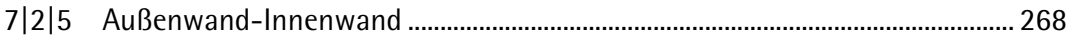

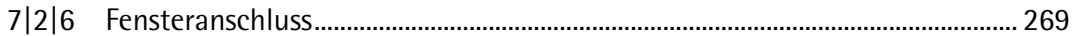

$7|2| 7 \quad$ Besonderheiten Nassbereich .................................................................................. 270

7|2|8 Besonderheiten bei brandabschnittsbildenden Bauteilen ................................. 270

$7|2| 8 \mid 1 \quad$ Anschluss der brandabschnittsbildenden Trennwand zur Außenwand ........271

$7|2| 8 \mid 2 \quad$ Anschluss der brandabschnittsbildenden Trenndecke zur Außenwand ........271

$7|2| 8 \mid 3 \quad$ Anschluss der brandabschnittsbildenden Trennwand zur Decke .....................272

$7|2| 8 \mid 4 \quad$ Anschluss der brandabschnittsbildenden Trennwand zum Dach......................272

7|3 Zusammenhang zwischen Komplexität und Kosten ......................................................... 273

$7|3| 1 \quad$ Vorstehender Balkon ............................................................................................... 273

$7|3| 2$ Rückspringendes Geschoß - Terrassenausgang ................................................... 273

8 Ausführung und Vorfertigung ...................................................................................... 275

8|1 Entwicklung der Ausführung .......................................................................................... 275

8|2 Systeme und deren Vorfertigungsgrad ......................................................................... 279

8|2|1 Holzskelettbau ......................................................................................................... 279

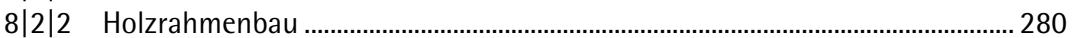

8|2|3 Massivholzbau............................................................................................................ 281

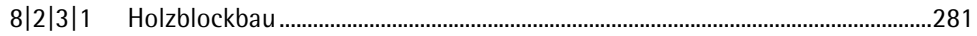

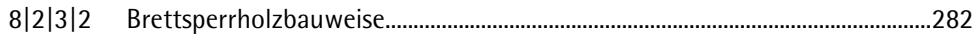

8|3 Schnittstellen in der Vorfertigung ............................................................................... 284

$8|3| 1 \quad$ Vorfertigung ohne Übergriff im Gewerk ................................................................ 285

$8|3| 2$ Vorfertigung mit Gewerkübergriff ............................................................................. 285

8|3|3 Transportfähigkeit im Rahmen der Vorfertigung ................................................ 287

8|4 Planungsverhalten im modernen Holzbau ............................................................................. 289

8|4|1 Technische Anforderungen .......................................................................................... 290

8|4|2 Leitungsführung/Installationen/Durchdringungen/Schächte............................ 292

8|4|3 Luftdichtheit........................................................................................................... 294

8|5 Ablauf von Vorfertigung und Montage............................................................................ 294

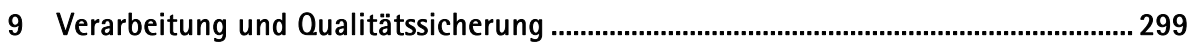

9|1 Holz, ein Baustoff mit besonderen Anforderungen ........................................................... 299

9|1|1 Egalisierung oder Optimierung ................................................................................... 299

9|1|2 Qualität bewerten - Holz verstehen ..................................................................... 300

9|1|3 Verarbeitungs- und Qualitätsmaßstäbe im Wandel der Zeit ............................. 300

9|2 Dauerhaftigkeit von Holzkonstruktionen ........................................................................... 301

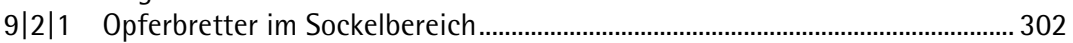

9|2|2 Wahl der Einbaufeuchte ........................................................................................ 303

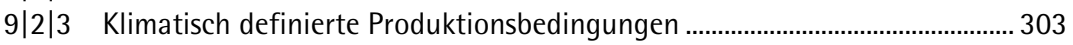

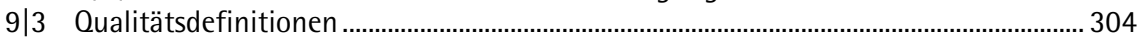


9|3|1 Die österreichischen Holzhandelsusancen 1973 _.................................................304

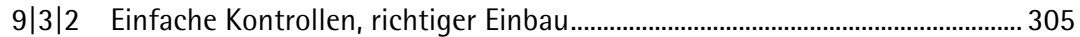

9|3|3 Ebenheit von Holzteilen ........................................................................................... 306

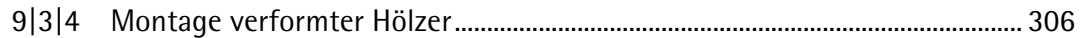

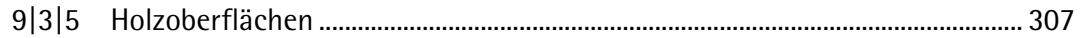

9|3|6 Offenheit bei Information ...................................................................................... 308

$9|3| 7$ Überlängen bei Schnittholzlieferungen ............................................................... 308

9|3|8 Metallspäne verursachen Fleckenbildung............................................................ 308

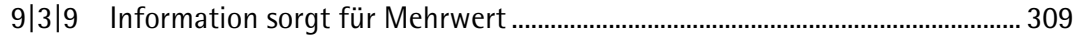

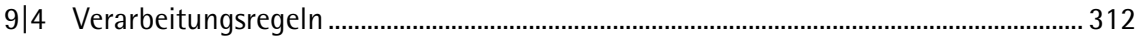

9|4|1 ÖNORM B 2320 Wohnhäuser aus Holz............................................................... 312

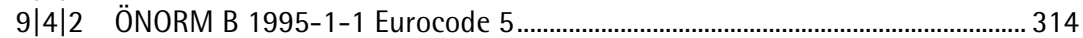

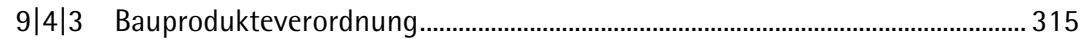

9|4|4 ÜA-Zeichen - Vorläufer der CE-Kennzeichnung ...................................................316

9|4|5 Eigen- und Fremdüberwachung ……………….................................................... 317

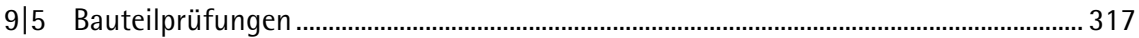

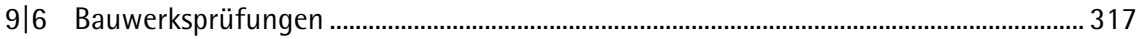

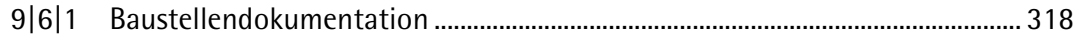

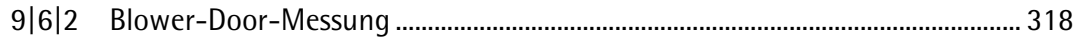

9|6|3 Gebäudethermografie................................................................................. 319

9|6|4 Kombinationsdiagnostik Blower-Door-Messung und Thermografie .............. 319

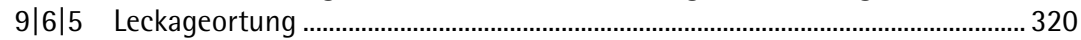

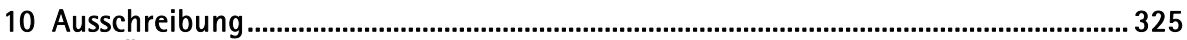

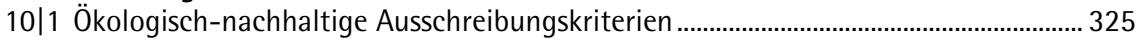

10|1|1 Implementierung ökologischer Aspekte................................................................ 325

$10|1| 1 \mid 1 \quad$ Zuschlagskriterien ....................................................................................................326

10|1|1|2 Auftragsausführungsklauseln ................................................................................326

$10|1| 1 \mid 3 \quad$ Technische Spezifikationen der Leistung …………………………………….....327

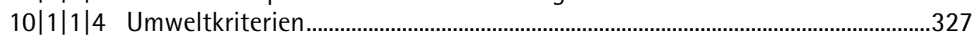

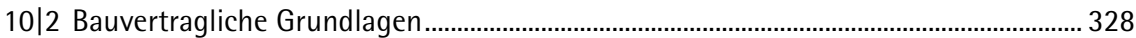

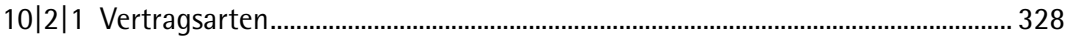

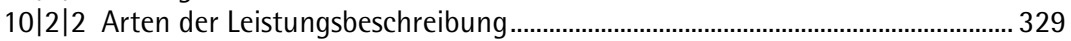

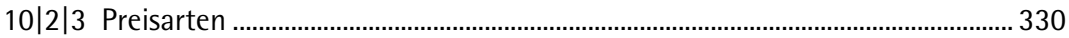

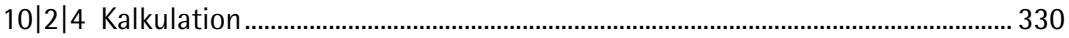

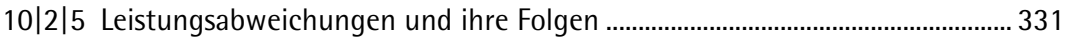

10|2|6 Dokumentation - „Wer schreibt, der bleibt" ...................................................... 333

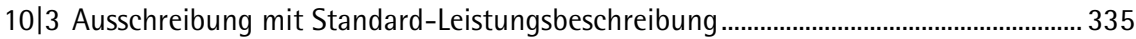

$10|3| 1$ Struktur und Inhalt der LB Hochbau LG 36 Holzbau ..........................................336

10|3|2 Leistungsbeschreibung Hochbau LG 36 Allgemeines ...........................................336

$10|3| 2 \mid 1$ Wählbare Vorbemerkungen ....................................................................................336

10|3|2|2 Sonderkosten der Baustelle ................................................................................337

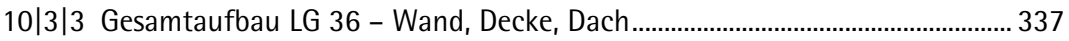

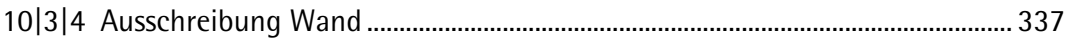

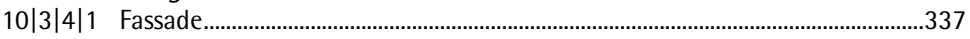

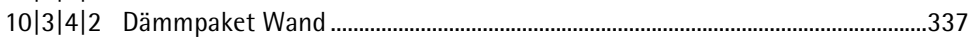

$10|3| 4 \mid 3 \quad$ Rohbauelement Holzrahmenwand .....................................................................338

$10|3| 4 \mid 4$ Rohbauelement Holzmassivwand ...........................................................................338

$10|3| 4 \mid 5 \quad$ Rohbauelement konventionell..............................................................................338

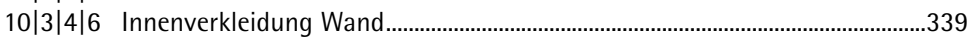

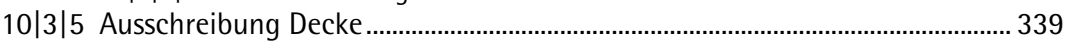

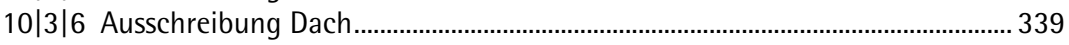

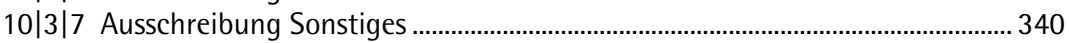

10|3|7|1 Holztragwerke Einzelbauteil......................................................................................340

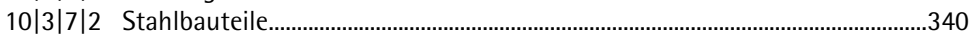

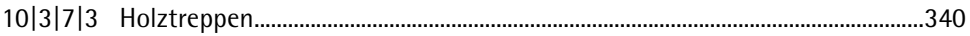


$10|3| 7 \mid 4$ Balkonkonstruktion und Geländer aus Holz .......................................................340

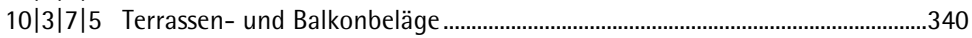

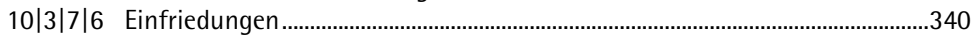

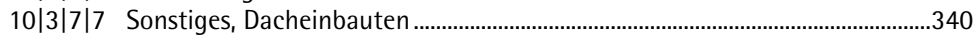

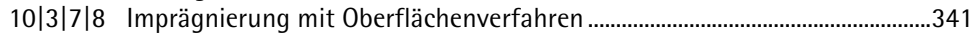

10|3|7|9 Imprägnierung mit Kesseldruckverfahren ...........................................................341

10|3|8 Beispiele für die Ausschreibungen von Holzbauleistungen............................... 341

10|3|8|1 Beispiel 1: Wandelement gemäß Bauteilkatalog „dataholz" ............................341

$10|3| 8 \mid 2$ Beispiel 2: Kalkulation Wandelement..................................................................342

$10|3| 8 \mid 3$ Beispiel 3: Dachkonstruktion ..............................................................................343

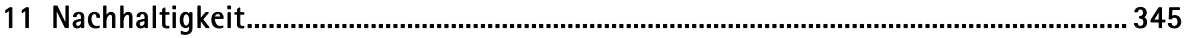

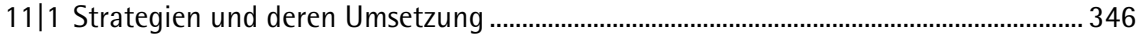

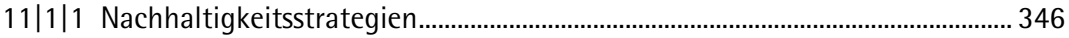

11|1|2 Implementierung ökologischer Aspekte in die Projektphasen .............................347

$11|1| 2 \mid 1 \quad$ Null-Phase, Vorprojekt, Machbarkeitsstudie..........................................................348

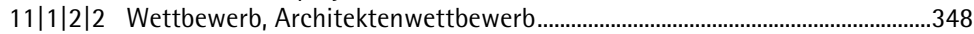

$11|1| 2 \mid 3$ Vorentwurf, Entwurf, Genehmigungsplanung ..................................................348

$11|1| 2 \mid 4$ Ausführungsplanung, Leistungsbeschreibungen, Vergabe................................349

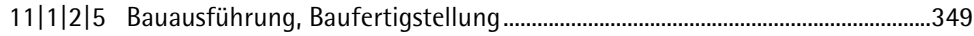

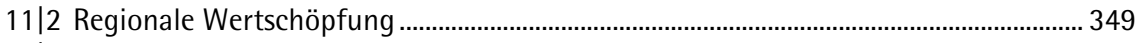

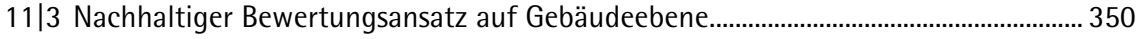

$11|3| 1$ Europäische Normung zum nachhaltigen Bauen ................................................... 353

11|3|1|1 ÖNORM EN 15978 [226]: Nachhaltigkeit von Bauwerken - Bestimmung der

Umweltleistung von Gebäuden - Berechnungsmethode .................................355

$11|3| 1 \mid 2$ ÖNORM EN 15804 [215]: Nachhaltigkeit von Bauwerken -

Umweltproduktdeklarationen - Grundregeln für die Produktkategorie

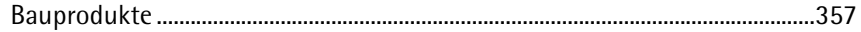

$11|3| 2$ Zertifizierungssysteme für Gebäude ....................................................................... 358

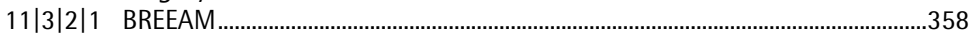

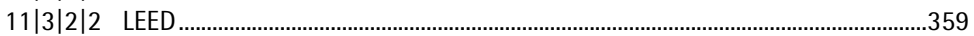

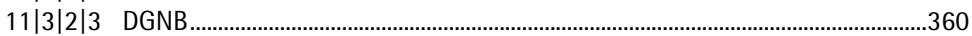

$11|3| 2 \mid 4$ Bewertungssystem Nachhaltiges Bauen für Bundesgebäude (BNB) ..............360

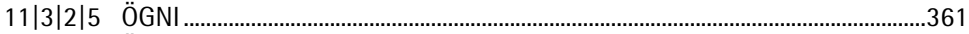

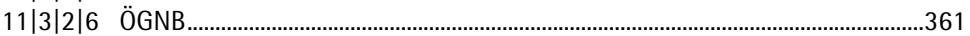

$11|3| 2 \mid 7 \quad$ klima:aktiv .............................................................................................................362

$11|3| 2 \mid 8$ Vergleichender Überblick der Gebäudezertifizierungssysteme..........................362

11|4 Nachhaltigkeitsbewertung von Schadstoffen aus Holz- und Holzwerkstoffen ......... 364

11/5 Holz als $\mathrm{CO}_{2}$ - und Energiespeicher .........................................................................................366

11|6 Ökobilanzierung von Baustoffen und Gebäuden ..................................................................369

$11|6| 1$ Festlegung des Ziels und des Untersuchungsrahmens.........................................370

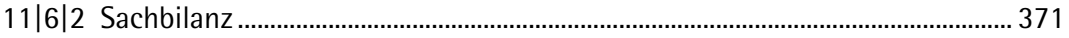

$11|6| 3$ Wirkungsabschätzung ……………………………………………………………... 371

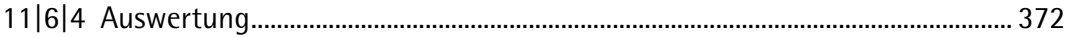

11|6|5 Infoplattformen............................................................................................. 372

11|7 Beispiel TQ-Bewertung - Plusenergieverbund Reininghaus Süd | TQB/Klimaaktiv ... 373

Quellennachweis.

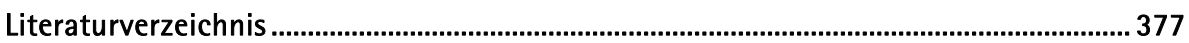

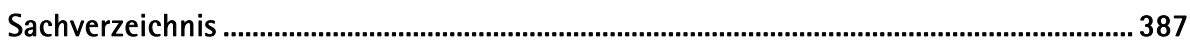

Autoren 